\title{
Precise Measurement of the Absolute Yield of Fluorescence Photons in Atmospheric Gases
}

\author{
AIRFLY Collaboration: M. Ave ${ }^{\mathrm{a}}$, M. Boháčovábc ${ }^{\text {, K. Daumiller }}{ }^{\mathrm{a}}$, P. Di Carlo ${ }^{\mathrm{d}}$, C. Di Giulio ${ }^{\mathrm{e}}$, \\ P. Facal San Luis ${ }^{\text {* }}$, D. Gonzales ${ }^{f}$, C. Hojvat ${ }^{g}$, J. R. Hörandel ${ }^{\text {h }}$, M. Hrabovskýi ${ }^{i}$, M. Iarlori ${ }^{\mathrm{d}}$, \\ B. Keilhauer ${ }^{\mathrm{a}}$, H. Klages ${ }^{\mathrm{a}}$, M. Kleifges ${ }^{\mathrm{j}}$, F. Kuehn ${ }^{\mathrm{g}}$, M. Monasor ${ }^{\mathrm{b}}$, L. Nožka ${ }^{\mathrm{c}}$, M. Palatka ${ }^{\mathrm{c}}$, S. Petrera ${ }^{\mathrm{d}}$, \\ P. Privitera ${ }^{b}, J$. Ridkyc ${ }^{c}$, V. Rizi ${ }^{d}$, B. Rouillé d'Orfeuil ${ }^{b}$, F. Salamida ${ }^{a}$, P. Schovánek ${ }^{c}$, R. Śmida $^{a}$, \\ H. Spinkak ${ }^{k}$ A. Ulrich ${ }^{1}$, V. Verzi ${ }^{\mathrm{e}}$, C. Williams ${ }^{\mathrm{b}}$

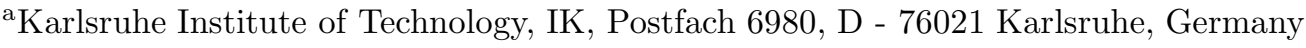 \\ ${ }^{\mathrm{b}}$ University of Chicago, Enrico Fermi Institute \& Kavli Institute for Cosmological Physics, \\ 5640 S. Ellis Ave., Chicago, IL 60637, USA \\ 'Institute of Physics of the Academy of Sciences of the Czech Republic, \\ Na Slovance 2, CZ-182 21 Praha 8, Czech Republic \\ dDipartimento di Fisica dell'Università de l'Aquila and INFN, Via Vetoio, I-67010 Coppito, Aquila, Italy \\ eDipartimento di Fisica dell'Università di Roma Tor Vergata and Sezione INFN, \\ Via della Ricerca Scientifica, I-00133 Roma, Italy \\ ${ }^{\mathrm{f}}$ Karlsruhe Institute of Technology, IEKP, Postfach 3640, D - 76021 Karlsruhe, Germany \\ ${ }^{\text {g}}$ Fermi National Accelerator Laboratory, Batavia, IL 60510, USA \\ hIMAPP, Radboud University Nijmegen, 6500 GL Nijmegen, The Netherlands \\ iPalacky University, RCATM, Olomuc, Czech Republic \\ ${ }^{j}$ Karlsruhe Institute of Technology, IPE, Postfach 3640, D - 76021 Karlsruhe, Germany \\ kArgonne National Laboratory, Argonne, IL 60439, USA \\ ${ }^{1}$ Physik Department E12, Technische Universität Muenchen, \\ James Franck Str. 1, D-85748 Garching, Germany
}

We have performed a measurement of the absolute yield of fluorescence photons at the Fermilab Test Beam. A systematic uncertainty at $5 \%$ level was achieved by the use of Cherenkov radiation as a reference calibration light source. A cross-check was performed by an independent calibration using a laser light source. A significant improvement on the energy scale uncertainty of Ultra-High Energy Cosmic Rays is expected.

\section{Introduction}

Fluorescence detection of Ultra High Energy Cosmic Rays (UHECRs) is a well established technique, pioneered by Fly's Eye [1], and today

*Corresponding author: facal@kicp.uchicago.edu an integral part of the Pierre Auger [2] and the Telescope Array 3 experiments. Excitation of the atmospheric nitrogen by the charged particles in the extensive air shower induces the emission of fluorescence photons, mostly in the 300$400 \mathrm{~nm}$ range. A Fluorescence Detector (FD) 
records this radiation to infer the cosmic ray energy and the particle type. For this purpose, the fluorescence light yield from the charged particles in the shower must be known for every emission point along the shower axis. A correction is then applied to account for atmospheric effects between the shower and the telescope, enabling an accurate, quasi-calorimetric, primary energy determination.

The uncertainty on the fluorescence light yield is one of the main systematic uncertainties on the cosmic ray energy determination by experiments that employ the fluorescence technique (e.g. $14 \%$ over a total $22 \%$ uncertainty for the Pierre Auger experiment). The AIRFLY collaboration has already performed a very precise measurement of the fluorescence spectrum and its pressure dependence [4], as well as the dependence of the emission on the temperature and humidity [5. AIRFLY measurements over electron kinetic energies ranging from $\mathrm{keV}$ to $\mathrm{GeV}$ using several accelerators have also proven the proportionality of the fluorescence yield with the electron energy deposit [6].

The final step in the precise characterization of the nitrogen fluorescence light emission is the measurement the absolute value of the yield for the main emission line at $337 \mathrm{~nm}$. The AIRFLY strategy to reduce the systematic uncertainties is to calibrate the experimental apparatus in situ, using photons emitted by a well know process: Cherenkov radiation [7. A second calibration method, with nearly independent systematic uncertainty, is based an absolutely calibrated laser light source. In this paper, we present preliminary results from a series of dedicated measurements at the Fermi National Accelerator Laboratory (Fermilab).

\section{Experimental method}

The measurements were performed at the Fermilab Test Beam Facility. Most of the measurements were carried out using the $120 \mathrm{GeV}$ proton beam, extracted from the Main Injector. Secondary beams of $32 \mathrm{GeV}$ pions and $8 \mathrm{GeV}$ positrons were also used.

A sketch of the apparatus is shown in Fig.

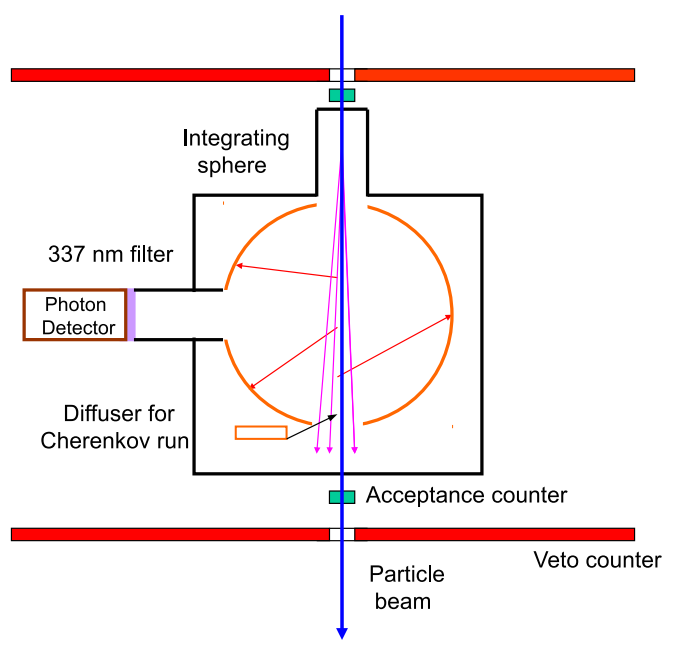

Figure 1. Layout of the experimental apparatus used at the Fermilab Meson Test Beam

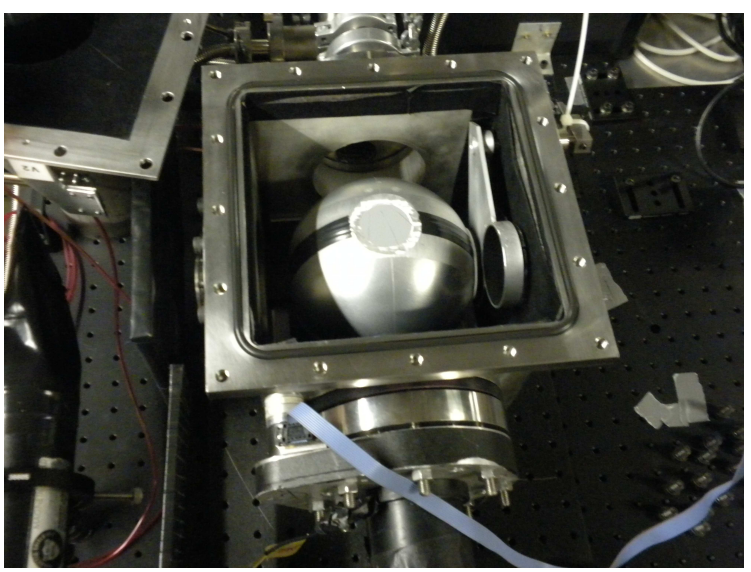

Figure 2. AIRFLY chamber with the integrating sphere fitted inside. 


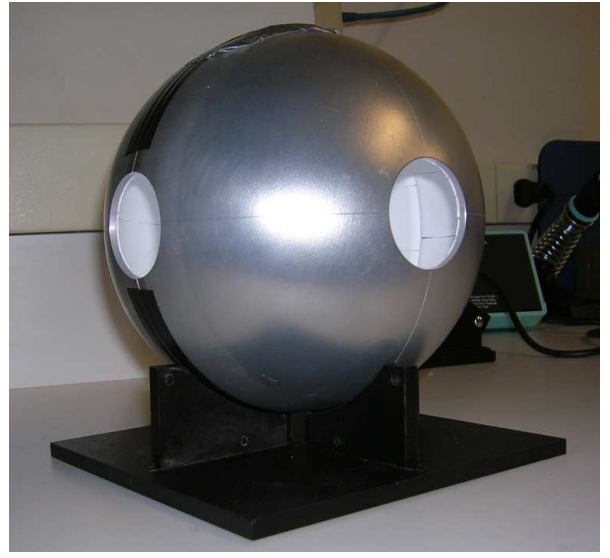

Figure 3 . The integrating sphere used in the measurements. The diffusive coating applied to the interior can be seen through two of the ports.

1. A fluorescence chamber made of a $3 \mathrm{~mm}$ thick stainless steel was placed in the beam path, with the corresponding flanges for windows, shutters, gauges, gas inlet and pump-out. Both the entrance and exit windows are $0.1 \mathrm{~mm}$ thick aluminum, with the entrance window before a $18 \mathrm{~cm}$ long tube to provide additional length for Cherenkov light production. An integrating sphere was used to collect light produced inside the chamber (Fig. 2). One of the ports of the sphere was open to a a gas-tight window fitted with a $337 \mathrm{~nm}$ filter and then coupled to the photon detector. A Hamamatsu H7195P photomultiplier (PMT) tube, with good single photoelectron resolution, was used for photon detection. The optical field of view was defined by a $40 \mathrm{~mm}$ diameter acceptance cylinder placed between the integrating sphere's port and the filter, and by circular apertures of the same size placed in front of the PMT photocathode. A mechanical shutter remotely controlled allowed to take background measurements. The chamber was internally covered with a black UV-absorbing material to avoid stray light.

The purpose of the integrating sphere is twofold: it increases the light collection solid an- gle (and thus maximizes the signal to noise ratio) and it works as equalizer of the detection efficiency of the set-up for the isotropic fluorescence light and for the highly directional Cherenkov light. The integrating sphere (Fig. 3) was built from two hollow aluminum half spheres coated with a material of very high diffusive reflectance. Light produced inside the sphere is collected over $4 \pi$ solid angle and at the same time isotropized by several diffusion bounces, so that the lambertian light output is independent of the original light distribution. A total of 4 ports were machined in the sphere, one was the detection port, two were for the beam entrance and exit, and the last one was opened at the top of the sphere. Two remotely controlled shutters could close the top and exit ports of the integrating sphere with plugs coated with the same diffusive material material using inside the sphere. With the exit port closed the Cherenkov is diffused back into the sphere and can reach the photon detector. With the exit port open the Cherenkov is absorbed by the chamber lining and thus only fluorescence can reach the photon detector. The top port compensates the open/closed position of the exit port to maintain always three open ports and thus the optical characteristics of the sphere. $\mathrm{i}$

The whole chamber was airtight and a remotely controlled system for gas and vacuum handling was used. Pure nitrogen and a dry-air mixture were used for the measurements and additionally helium and argon were used for background runs. The pressure, temperature and humidity inside the chamber were monitored remotely using the appropriate sensors.

A set of particle counters was used for beam monitoring. At the entrance of the chamber a 10 $\mathrm{mm}$ diameter finger counter was used for beam tagging. At the exit the beam was tagged with a Cherenkov counter, a $10 \mathrm{~mm}$ diameter cylindrical rod made of UV-transparent acrylic material. The rod was $30 \mathrm{~mm}$ long and allowed very good single particle resolution with fast timing. Before and after the chamber two big scintillator pads with a central $10 \mathrm{~mm}$ diameter hole for beam passage provided a veto for off-axis particles. The chamber and the counters were mounted on an optical breadboard for precise mounting and 
alignment, and placed on a remotely movable table that allowed the alignment of the apparatus with the beam by maximizing the finger and Cherenkov counters rates. The beam profile was monitored by wire chambers placed before and after the AIRFLY apparatus and was typically 3 $\mathrm{mm} \times 4 \mathrm{~mm}$ wide.
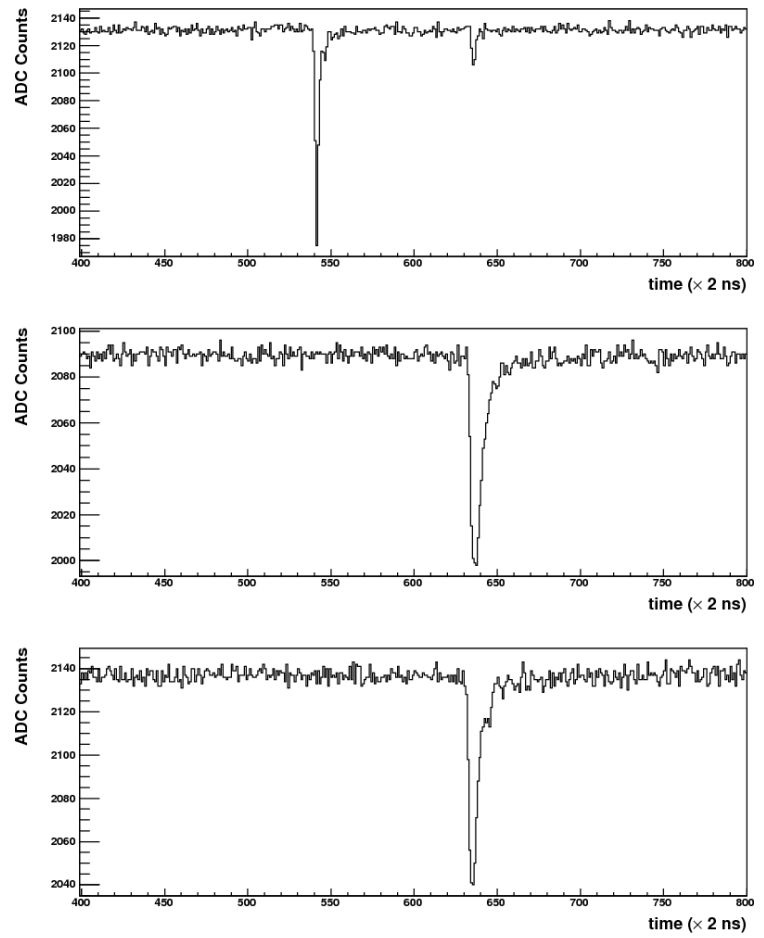

Figure 4. The signal of a train of bunches for the Cherenkov rod (top plot) and the two veto counters (middle and bottom plots). Two particles in two different bunches can be observed: the first one passes through the rod while the second one passes through both veto counters (and leaves also a small signal in the rod when it hits the PMT glass producing a small amount of Cherenkov in it).

The trigger and DAQ were designed considering the characteristics of the beam timing: within a $4 \mathrm{~s}$ beam spill, particles were grouped in trains of bunches. Trains were separated in time by 10 $\mu s$, and bunches within a train were separated by 19 ns. Typical conditions for data taking with the proton beam were 30 bunches per train, and a multiplicity of $2 \cdot 10^{5}$ particles per spill. The trigger logic was built from the coincidence of a train trigger gate, issued in correspondence of the arrival of each train of bunches, and a single particle trigger gate, coming from a beam monitoring scintillator counter. Both triggers were provided by the Test Beam Facility. Whenever a trigger was issued the signals from the scintillator counters and photomultipliers were digitized by a 12 bit $500 \mathrm{MHz}$ FADC and 600 samples (equivalent to $1.2 \mu \mathrm{s}$, containing the entire train of bunches) were saved in the FADC memory. The data for the whole spill was stored in the FADC internal memory and then readout and saved to disk in about $40 \mathrm{~s}$ between spills. As an example, the ADC trace of the beam counters for one trigger is shown in Fig. 4 .

Data during the test beam was taken in a different configurations. For each configuration runs of up to half hour (i.e. 30 spills) of data were acquired. Runs in the same conditions were repeated periodically to improve statistics and to assure redundancy and consistency.

\section{Data analysis and results}

Offline data analysis for one run proceeds selecting single particles that cross the fiducial volume of the camera, hence are tagged by the beam counters placed at the entrance and the exit of the chamber (Fig. 5). For these selected particles we require that no signal is present in any of the veto counters. Additional analysis cuts are placed to discard trains that have unusually large number of particles passing through the veto counters, as this has been shown to improve the background conditions without excessively penalizing statistics. Once the clean single protons have been selected the PMT signal is analyzed and the photons in coincidence with the selected protons are counted (Fig 6). The signal $S$, in units of photoelectrons per beam particle, pbp, is then calculated. 


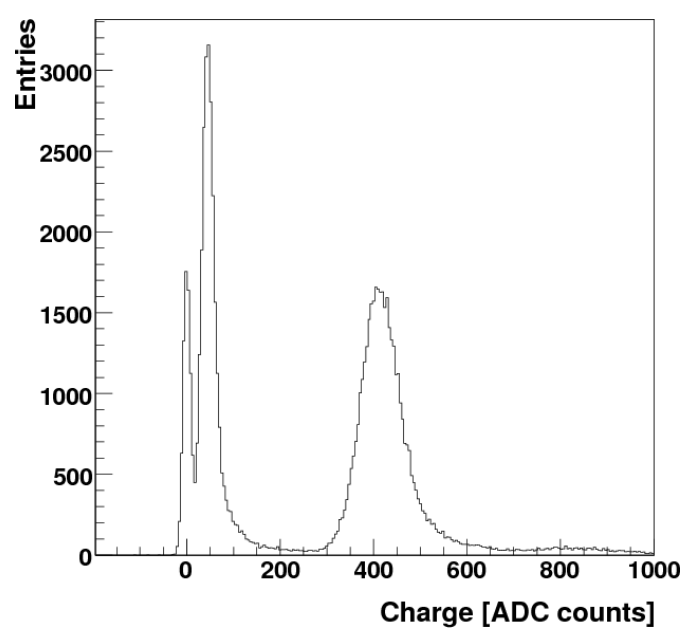

Figure 5. Spectrum of the Cherenkov rod. From left to right the three peaks correspond to pedestal, particles hitting the PMT window and single particles passing through the Cherenkov radiator. The fourth rightmost smaller peak corresponds to two particles in the same bunch.

The measured signal in the a fluorescence measurement taken in a given gas, $\mathrm{S}_{F L}^{g a s}($ meas), is given by:

$S_{F L}^{g a s}($ meas $)=S_{F L}^{g a s}+B_{F L}^{g a s}$,

where $\mathrm{S}_{F L}^{g a s}$ is the signal from the $337 \mathrm{~nm}$ band, and $\mathrm{B}_{F L}^{g a s}$ is attributable to background. The best determination of the overall background can be obtained combining measurements from different gases. From Eq. 11, the difference of the measured signal in $\mathrm{N}_{2}$ and air is given by:

$\Delta S_{F L}=S_{F L}^{\mathrm{N}_{2}}-S_{F L}^{\text {air }}+B_{F L}^{\mathrm{N}_{2}}-B_{F L}^{\mathrm{air}}$.

Since the beam related background and the primary interactions are the same and secondary particle production is very similar in nitrogen and air backgrounds cancel in Eq. 2. Thus,

$\Delta S_{F L}=S_{F L}^{\mathrm{N}_{2}}\left(1-\frac{1}{r_{\mathrm{N}_{2}}}\right)$,

where $r_{\mathrm{N}_{2}}$ is the ratio of the $337 \mathrm{~nm}$ fluorescence in pure nitrogen to the signal in air. This ratio

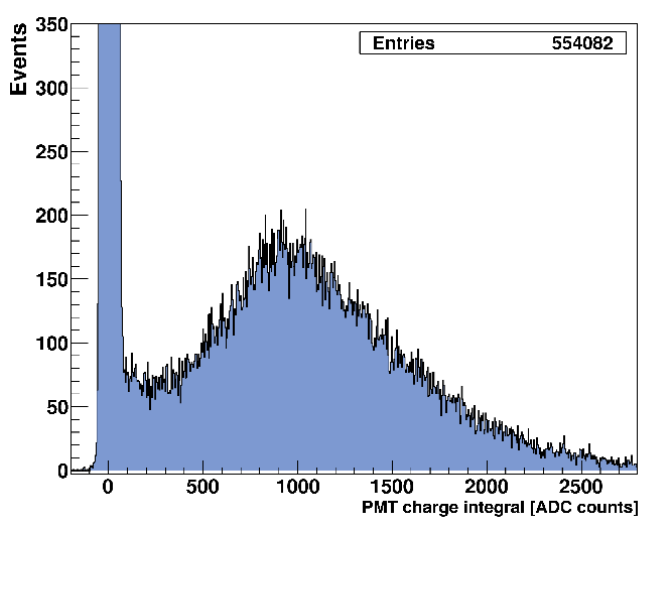

Figure 6. PMT spectrum for a run. The events inside the single photoelectron peak are counted to define the signal.

was measured by AIRFLY as a function of pressure in 4 and was cross-checked in our Fermilab experimental apparatus using a ${ }^{241} \mathrm{Am}$ radioactive source, giving $7.45 \pm 0.10$ at $1000 \mathrm{hPa}$.

We measure $\Delta S_{F L}=(16.83 \pm 0.13) \cdot 10^{-4} \mathrm{pbp}$. From Eq. 3, we derive the background-subtracted fluorescence signal:

$S_{F L}^{\mathrm{N}_{2}}=(19.44 \pm 0.15) \cdot 10^{-4} \mathrm{pbp}$,

with the background (Eq. 1) estimated as a 3\% of the signal.

In the Cherenkov calibration measurement, both Cherenkov and fluorescence emission in the gas contribute to the measured signal:

$S_{C H}^{g a s}($ meas $)=S_{F L}^{g a s}+S_{C H}^{g a s}+B_{F L}^{g a s}+B_{C H}$,

where $S_{C H}^{g a s}$ is the signal from Cherenkov light in the $337 \mathrm{~nm}$ band emitted in the gas under study, and $B_{C H}$ takes into account background originating from the interaction of the beam particles in the exit port plug (that is independent of the gas filling the chamber). $B_{C H}$ can be estimated directly from the difference in the vacuum measurement in fluorescence and Cherenkov modes

$B_{C H}=(2.63 \pm 0.23) \cdot 10^{-4} \mathrm{pbp}$, 
a value that is $\sim 10 \%$ of the Cherenkov signal. We made several cross-check of this background, for example changing the exit port plug material to a thin Mylar foil where no light production is expected.

We measure $S_{C H}^{\mathrm{N}_{2}}$ (meas $)=(32.89 \pm 0.15)$. $10^{-4}$ pbp. Using Eq. [5 we obtain

$S_{C H}^{\mathrm{N}_{2}}=(10.27 \pm 0.23) \cdot 10^{-4} \mathrm{pbp}$,

and from it and Eq. 4 we derive

$R^{\mathrm{N}_{2}}=\frac{S_{F L}^{\mathrm{N}_{2}}}{S_{C H}^{\mathrm{N}_{2}}}=1.893 \pm 0.045$,

the ratio of fluorescence to Cherenkov $337 \mathrm{~nm}$ photons produced inside the chamber.

In order to derive the absolute $337 \mathrm{~nm}$ yield in air, we performed a full Monte Carlo simulation of the setup, where all individual components are simulated according to measurements done in the laboratory. The absolute fluorescence yield in the simulation, $Y_{M C}^{\text {air }}$, which determines a corresponding expected Fluorescence/Cherenkov ratio $R_{M C}^{a i r}$, can be scaled to match the measured value of Eq. 8 and obtain our measurement of the absolute fluorescence yield in air:

$Y^{\text {air }}=5.60 \pm 0.13 \gamma_{337 \mathrm{~nm}} / \mathrm{MeV}$,

where the uncertainty is statical only. Our preliminary estimate for the systematic uncertainty of the measurement is better than $5 \%$, dominated by the uncertainty in the wavelength dependence of the PMT quantum efficiency and the 337 filter transmission, accounting for the different spectral distributions of the fluorescence and Cherenkov signals. The detailed analysis of the uncertainties is still, however, ongoing.

In the laser calibration method, we use a $337 \mathrm{~nm}$ nitrogen laser and an NIST calibrated probe to determine the overall efficiency of the set-up, the number of detected photoelectrons per photon entering the sphere. Using this efficiency, the number in Eq. 4 and a detailed Monte Carlo simulation of the laser calibration the value

$Y_{\mathrm{LASER}}^{\mathrm{air}}=5.56 \pm 0.07 \gamma_{337 \mathrm{~nm}} / \mathrm{MeV}$,

is obtained, which has an uncertainty nearly independent of the one from the Cherenkov calibration. The systematic uncertainty is dominated by the $5 \%$ uncertainty in the absolute calibration of the laser probe.

\section{Outlook}

The preliminary results on the absolute fluorescence yield reported in this work are compatible with the current values used in Fluorescence Detector analysis (see Ref. [8] for a review of the different measurements.) We expect a final measurement with a systematic uncertainty below $5 \%$, a significant improvement over previous measurements, which will correspondingly improve the uncertainty on the energy scale of UHECR measurements.

\section{REFERENCES}

1. R. M. Baltrusaitis et al., Nucl. Instrum. Meth. A 240 (1985) 410.

2. J. A. Abraham et al. [The Pierre Auger Collaboration], Nucl. Instrum. Meth. A 620 (2010) 227 arXiv:0907.4282 [astro-ph.IM]].

3. Y. Tameda et al., Nucl. Instrum. Meth. A 609 (2009) 227.

4. M. Ave et al. [AIRFLY Collaboration], Astropart. Phys. 28 (2007) 41 arXiv:astro-ph/0703132.

5. M. Ave et al. [AIRFLY Collaboration], Nucl. Instrum. Meth. A 597 (2008) 50 arXiv:0711.4583 [astro-ph]].

6. M. Ave et al. [AIRFLY Collaboration], Nucl. Instrum. Meth. A 597 (2008) 46.

7. M. Bohacova et al. [AIRFLY Collaboration], Nucl. Instrum. Meth. A 597 (2008) 55 arXiv:0812.3649 [astro-ph]].

8. J. Rosado, F. Blanco and F. Arqueros, Astropart. Phys. 34 (2010) 164 arXiv:1004.3971 [astro-ph.IM]]. 